Sādhanā Vol. 40, Part 3, May 2015, pp. 961-972. (C) Indian Academy of Sciences

\title{
High Reynolds number liquid layer flow with flexible walls
}

\author{
J S B GAJJAR \\ School of Mathematics, University of Manchester, Manchester, M13 9PL, UK \\ e-mail: j.gajjar@manchester.ac.uk
}

MS received 13 May 2014; accepted 6 November 2014

\begin{abstract}
The stability of liquid layer flow over an inclined flexible wall is studied using asymptotic methods based on the assumption that the Reynolds number is large. The flexible wall behaviour is described by a spring-plate model, and parameters chosen so that the wall flexibility affects the governing boundary layer problem. For the case of a rigid wall, the problem reverts to one studied by Gajjar. Asymptotic analysis of the governing equations leads to the triple-deck equations governing the interaction between the wall layer and the free-surface. The linearised and other solution properties of these set of equations are discussed.
\end{abstract}

Keywords. Stability; triple-deck; separation; boundary-layer; compliant wall.

\section{Introduction}

The stability of liquid layer flow over a rigid inclined wall has been extensively studied by many people including papers by Yih $(1955,1963,1965)$, Benjamin (1957) amongst others. The main conclusion stemming from these studies is that the fully developed half-Poiseuille flow becomes unstable to long wavelength disturbances at a critical Reynolds number of $R=R_{c}=5 / 6 \cot \beta^{*}$, where $\beta^{*}$ is the inclination of the wall to the horizontal. There are of course many other studies which have examined the nonlinear development of disturbances and other aspects as well.

The problem studied here is the liquid layer flow over an inclined flexible wall, based on the assumption that the Reynolds number is large. The main primary motivation for our study is to understand the effect of boundary-layer separation over flexible walls such as that schematically depicted in figure 1 in which an oncoming boundary-layer flow separates due to some surface obstacle and behind the obstacle we have a region in which the wall is flexible. Such configurations have potential application to aerodynamic and marine flows. Boundary-layer flows over flat plates have been extensively studied and it is well-known that wall flexibility greatly affects the growth of Tollmien-Schlichting waves, see Carpenter \& Garrad (1985). The problem of a laminar separation bubble interacting with a flexible wall has received little or no attention and this provides a motivation for the current study. Provided the appropriate scalings are chosen, there is a great deal of similarity between the boundary-layer problem and the problem studied which involves a liquid layer flow. In fact, the governing equations for both flows under appropriate limits can be reduced to the triple-deck equations, but with slightly different pressure-displacement 


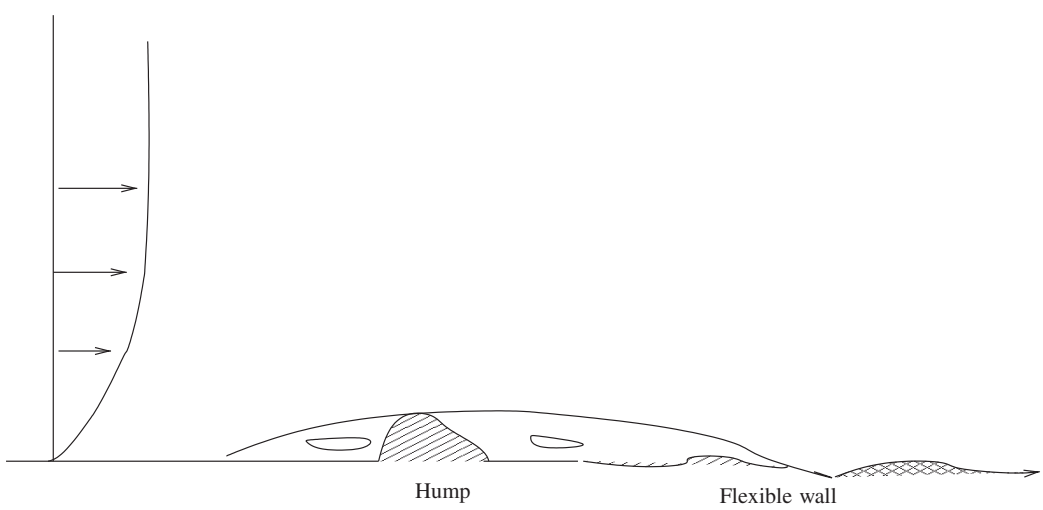

Figure 1. Boundary-layer separation induced by a hump with a flexible wall.

interaction laws. The liquid layer flow problem gives rise to an interaction law which is easier to handle numerically, and this is the reason why we have chosen to study this problem first, as it may offer some insight into the real problem of interest which is the boundary-layer flow over a flexible wall with flow separation present.

\section{Problem formulation and governing equations}

Consider the liquid layer flow over a flexible wall $z=h(x, t)$ with a free-surface $z=\eta(x, t)$, see figure 2 . We will assume that the wall motion is modelled by a standard spring-plate model as described in Carpenter \& Garrad (1985). The governing equations are the continuity and Navier-Stokes equations given by

$$
\begin{gathered}
u_{x}+w_{z}=0, \\
u_{t}+u u_{x}+w u_{z}=g \sin \beta^{*}-\frac{1}{\rho} p_{x}+v\left(u_{x x}+u_{z z}\right), \\
w_{t}+u w_{x}+w w_{z}=-g \cos \beta^{*}-\frac{1}{\rho} p_{z}+v\left(w_{x x}+w_{z z}\right) .
\end{gathered}
$$

Here $p$ is the pressure, $(u, w)$ are the velocity components in the $(x, z)$ directions with $z$ measured normal to the wall which is inclined at some angle $\beta^{*}$ far upstream, see figure $2, v$ the kinematic viscosity of the fluid, $\rho$ the fluid density and $g$ the acceleration due to gravity. The boundary conditions are those of continuity of stress at the interface $z=\eta(x, t)$. The kinematic condition

$$
w=\eta_{t}+u \eta_{x}
$$

also holds at $z=\eta(x, t)$. At the rigid-flexible wall $z=h(x, t)$ the stream-wise and normal velocity components satisfy

$$
u=0 \quad \text { on } \quad z=h(x, t), \quad w=\frac{\partial h}{\partial t} \quad \text { on } \quad y=h(x, t) .
$$

The wall motion is governed by the spring-plate model equation

$$
M h_{t t}+B h_{x x x x}-T h_{x x}+\kappa h=-\Delta p_{s},
$$




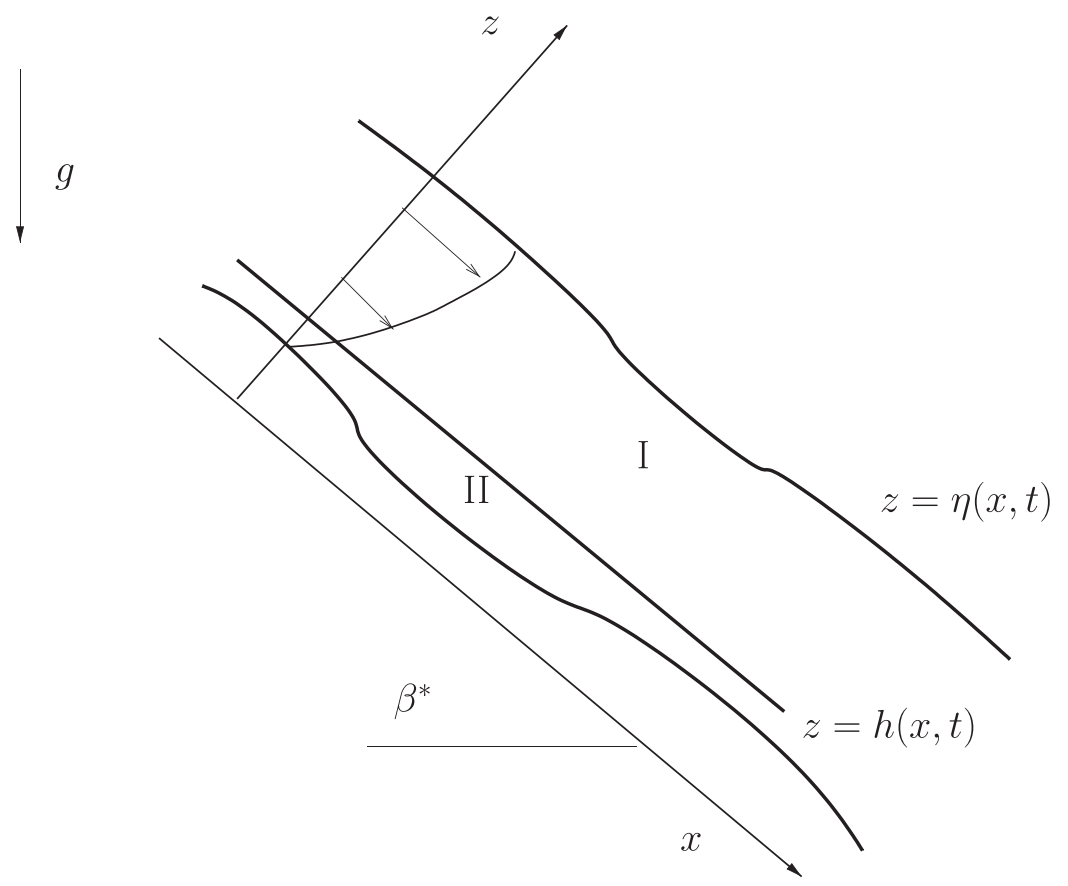

Figure 2. Schematic diagram showing the liquid layer flow over an inclined flexible surface $z=h(x, t)$ with a free-surface $z=\eta(x, t)$.

where $\Delta p_{s}$ denotes the change in mechanical fluid pressure due to the displacement of the flexible wall, $M$ is the plate mass density per unit length, $B$ the flexural rigidity of the plate, $T$ the tension and $\kappa$ the spring stiffness, see Carpenter \& Garrad (1985).

Far upstream we assume that flow is fully developed and steady with undisturbed depth $z=h_{0}$ and there is an exact solution of the governing equations given by

$$
\begin{gathered}
u=U_{B}^{*}(z)=\frac{g \sin \beta^{*}}{2 v}\left(h_{0}^{2}-\left(z-h_{0}\right)^{2}\right), \\
w=W_{B}^{*}=0, \\
p=P_{B}^{*}(z)=p_{a t m}-\rho g\left(z-h_{0}\right) \cos \beta^{*} .
\end{gathered}
$$

Here $p_{\text {atm }}$ is the pressure just above the liquid-air interface.

The governing equations are non-dimensionalised with respect to the basic velocity at the interface

$$
U_{c}=2 U_{B}^{*}\left(h_{0}\right)=\frac{g h_{0}^{2}}{v} \sin \beta^{*},
$$

and length scale $h_{0}$. Thus the following non-dimensional variables are introduced.

$$
\bar{x}=\frac{1}{h_{0}} x \quad ; \quad \bar{z}=\frac{1}{h_{0}} z \quad ; \quad \bar{h}=\frac{1}{h_{0}} h \quad ; \quad \bar{u}=\frac{1}{U_{c}} u \quad ;
$$


This introduces a Reynolds number $R e$ given by

$$
R e=\frac{U_{c} h_{0}}{v}=\frac{g h_{0}^{3} \sin \beta^{*}}{v^{2}} .
$$

To motivate the scalings used below, we first consider the steady case discussed by Gajjar (1987) where more details of the scaling arguments may be found. Consider free-surface perturbations of $O(\delta)$. We will assume that displacement of the free-surface causes induced change in hydrostatic pressure of $O(\Delta p)$. From the $\bar{z}$ momentum equation

$$
\bar{u} \bar{w}_{\bar{x}}+\bar{w} \bar{w}_{\bar{z}}=-\bar{p}_{\bar{z}}-\frac{1}{R e \tan \beta^{*}}+\frac{1}{R e}\left(\bar{w}_{\bar{x} \bar{x}}+\bar{w}_{\bar{z} \bar{z}}\right),
$$

we obtain

$$
\Delta p=O\left(\frac{\delta}{R e \tan \beta^{*}}\right) .
$$

Next, assume that the displacement of the free-surface induces a transverse pressure gradient of $O(\lambda)$, over a long length scale $L$ as in Smith (1977). Then $\lambda \sim \Delta p$ and from momentum balances

Hence

$$
\lambda \sim \frac{u \Delta w}{L} \sim \frac{\delta}{L^{2}}
$$

$$
\lambda \sim \frac{\delta}{\operatorname{Re} \tan \beta^{*}} \sim \frac{\delta}{L^{2}} .
$$

Next, viscous forces come into play when $\bar{z}=O(\delta)$ to reduce the slip velocity of $O(\delta)$ to zero. Viscous inertial balances give

$$
\frac{\delta^{2}}{L} \sim \frac{\lambda}{L} \sim \frac{\delta}{\operatorname{Re} \delta^{2}}
$$

Solving gives the important scalings, see Gajjar (1987)

$$
\delta \sim R e^{-2 / 7}, L \sim R e^{1 / 7}, \lambda \sim R e^{-4 / 7}, \tan \beta^{*} \sim R e^{-5 / 7} .
$$

If $\beta^{*} \ll R e^{-5 / 7}$ then the hydrostatic-induced pressure gradient is more important. If $\beta^{*} \gg$ $R e^{-5 / 7}$ the transverse pressure gradient is important. In the above we have ignored surface tension effects. However, these can be readily incorporated. Surface tension effects also come in at the same order provided

$$
C=O\left(\frac{\delta}{L^{2} \operatorname{Re} \Delta p}\right)=O\left(\frac{1}{\operatorname{Re}}\right) .
$$

where $C$ is the Capillary number

$$
C=\frac{U_{c} \mu}{\sigma}=\frac{\rho g h_{0}^{2} \sin \beta^{*}}{\sigma}
$$

With the above scalings, we introduce a small parameter $\epsilon=R e^{-1 / 7}$ and let $X=\epsilon \bar{x}$, and

$$
\tan \beta^{*}=\frac{\epsilon^{5}}{s}, \quad C=\epsilon^{7} \bar{C} .
$$

The free surface is expressed as

$$
\bar{z}=1+\epsilon^{2} \eta .
$$


and

$$
\bar{z}=\epsilon^{2} H(X)
$$

describes the wall with $H(X)$ being the reduced wall shape. As discussed in Gajjar (1987) there are two distinct asymptotic regions. In region I, see figure 2, where $z=O(1)$ the flow quantities have the expansions

$$
\bar{u}=U_{B}(\bar{z})+\epsilon^{2} u_{1}+\ldots, \quad \bar{w}=\epsilon^{3} w_{1}+\ldots, \quad \bar{p} \sim \epsilon^{2} \bar{s}(1-\bar{z})+\epsilon^{4} p_{1}+O\left(\epsilon^{6}\right),
$$

where $U_{B}=\bar{z}-\bar{z}^{2} / 2$ is the basic flow in terms of non-dimensional quantities and the $u_{1}$ term is the perturbation to the basic flow. Substitution into the continuity and Navier-Stokes Eqs. (1-3) gives

$$
\begin{gathered}
u_{1}=A(X) U_{B \bar{z}}, \quad w_{1}=-A^{\prime}(X) U_{B}, \\
p_{1}=P(X)+A^{\prime \prime}(X) \int_{0}^{\bar{z}} U_{B}^{2}(q) d q .
\end{gathered}
$$

Here, $A(X)$ is an unknown displacement function, and $P(X)$ is the disturbance pressure at the wall.

Applying the boundary conditions at the free-surface gives

$$
A(X)=-\eta(X), \quad P(X)=s \eta-A^{\prime \prime}(X) \gamma-\frac{1}{\bar{C}} \frac{d^{2} \eta}{d X^{2}},
$$

where

$$
\gamma=\int_{0}^{1} U_{B}^{2}(q) d q .
$$

The above solutions do not satisfy the full boundary conditions and a boundary-layer is needed. Next, introduce the boundary-layer of thickness $O\left(\epsilon^{2}\right)$ with $\bar{z}=\epsilon^{2} Z$ and there the flow quantities expand as

$$
\begin{gathered}
\bar{u} \sim \epsilon^{2} U_{1}+\ldots, \quad \bar{w} \sim \epsilon^{5} W_{1}+\ldots, \\
\bar{p} \sim \epsilon^{2} s+\epsilon^{4}\left(-s Z+P_{1}\right)+\ldots .
\end{gathered}
$$

After substitution into the Navier-Stokes equations we find the reduced governing equations as

$$
\begin{gathered}
U_{1 X}+W_{1 Z}=0 \\
U_{1} U_{1 X}+W_{1} U_{1 Z}=-P_{1 X}+U_{1 Z Z}, \\
P_{1 Z}=0
\end{gathered}
$$

with the boundary conditions

$$
\begin{gathered}
U_{1}=W_{1}=0 \quad \text { at } \quad Z=H(X), \\
U_{1} \rightarrow Z+A(X) \quad \text { as } \quad Z \rightarrow \infty, \\
U_{1} \rightarrow Z \text { as } \quad X \rightarrow-\infty,
\end{gathered}
$$

required for matching with the region $\mathrm{I}$ and far upstream where there are assumed to be no disturbances. The above problem was first formulated in Gajjar (1987) and linearised as well as nonlinear solutions are presented there for different wall shapes. See also Logue (2008).

Next, we will consider the same problem but now with the flexible wall present, and we will consider the time and other scalings such that the present solution structure remains largely 
intact, but with unsteadiness affecting mainly the wall layer. For unsteady effects to come in the boundary layer where $\bar{z}=O\left(\epsilon^{2}\right)$ we require a temporal scaling $t=\epsilon^{-3} T$. This introduces an extra $U_{T}$ term in the stream-wise momentum equation. Note that the wall motion is given by the spring-plate model which in unscaled variables is

$$
M h_{t t}+B h_{x x x x}-T_{e} h_{x x}+\kappa h=-\Delta p_{s} .
$$

Introduce the scalings

$$
\begin{gathered}
h=\epsilon^{2} H(X, T), \quad M=\epsilon^{-4} C_{M}, \\
B=\epsilon^{-2} B_{M}, \quad T_{e}=O(1), \quad \kappa=\epsilon^{2} K_{e},
\end{gathered}
$$

where the parameters $C_{M}, B_{M}, T_{e}, K_{e}$ are $O(1)$ quantities.

In summary, the governing equations become after setting $U=U_{1}, V=W_{1}$, :

$$
\begin{gathered}
U_{X}+V_{Z}=0 \\
U_{T}+U U_{X}+V U_{1 Z}=-P_{X}+U_{Z Z}, \quad P=P(X, T) .
\end{gathered}
$$

The boundary conditions are

$$
\begin{gathered}
U=0, \quad V=H_{T} \quad \text { on } \quad Z=H(X, T), \\
U \rightarrow Z+A(X, T) .
\end{gathered}
$$

The interaction law is now

$$
P=-s A-\left(\gamma-\frac{1}{\bar{C}}\right) A^{\prime \prime} .
$$

with the wall motion governed by

$$
C_{M} H_{T T}+B_{M} H_{X X X X}-T_{e} H_{X X}+K_{e} H=-P .
$$

The above Eqs. (15a, b, c, d, e, f) are the triple-deck equations for the nonlinear unsteady flow over a flexible wall.

It is noted that with appropriate choice of scalings, the boundary-layer flow over a hump with a flexible wall leads to exactly the same set of equations except that the interaction law (15e) is replaced by

$$
P=\frac{1}{\pi} \int_{-\infty}^{\infty} \frac{A^{\prime}(\xi, T)}{X-\xi} d \xi
$$

Given suitable initial conditions the problem (15a, b, c, d, e, f) may be solved numerically. The steady nonlinear problem leads to separated flow for various wall shapes. In what follows we restrict attention to the linearised version of $(15 \mathrm{a}, \mathrm{b}, \mathrm{c}, \mathrm{d}, \mathrm{e}, \mathrm{f})$, linearised about the undisturbed flow over a flat wall corresponding to the solution $U=Z, V=P=A=H=0$ and discuss the solution properties.

\section{Linear, unsteady analysis and properties of the dispersion relation}

Consider small disturbances about the basic state $U=Z, V=P=A=0$ and let

$$
\begin{gathered}
U(X, Z, T)=Z+\delta \tilde{U}(X, Z) e^{-i \omega T}+c . c, \quad V(X, Z, T)=\delta \tilde{V}(X, Z) e^{-i \omega T}+c . c, \\
P(X, T)=\delta \tilde{P}(X) e^{-i \omega T}+c . c ., \quad A(X, T)=\delta \tilde{A}(X) e^{-i \omega T}+c . c ., \\
H(X, T)=\delta \tilde{H}(X) e^{-i \omega T}+c . c,
\end{gathered}
$$


where $\delta \ll 1$ and c.c. denotes the complex conjugate.

We will also take Fourier Transforms (in $X$ ) defined by

$$
\tilde{U}^{*}(k, Z)=\int_{-\infty}^{\infty} \tilde{U}(X, Z) e^{-i k X} d X
$$

with the inverse

$$
\tilde{U}(X, Z)=\frac{1}{2 \pi} \int_{-\infty}^{\infty} \tilde{U}(k, Z) e^{i k X} d k .
$$

After substituting for the disturbances into Eqs. (15a, b, c, d, e, f) and linearising for $\delta$ small, we obtain the following system of equations and boundary conditions:

$$
\begin{gathered}
i k \tilde{U}^{*}+\tilde{V}_{Z}^{*}=0, \\
-i \omega \tilde{U}^{*}+i k Z \tilde{U}^{*}+\tilde{V}^{*}=-i k \tilde{P}^{*}+\tilde{U}_{Z Z}^{*}, \\
\tilde{U}^{*}=-\tilde{H}^{*}, \quad \tilde{V}^{*}=-i \omega H^{*} \quad \text { on } \quad Z=0, \\
\tilde{U}^{*} \rightarrow \tilde{A}^{*} \quad \text { as } \quad Z \rightarrow \infty \\
\tilde{P}^{*}=-\left(s-\left(\gamma-\frac{1}{C}\right) k^{2}\right) \tilde{A}^{*}, \\
-\tilde{P}^{*}=D_{a}(\omega, k) \tilde{H}^{*},
\end{gathered}
$$

where

$$
D_{a}(\omega, k)=-C_{M} \omega^{2}+B_{M} k^{4}+T_{E} k^{2}+K_{E}
$$

The equations can be solved in terms of Airy functions by introducing the variable $\xi=$ $(i k)^{1 / 3}\left(Z+\xi_{0}\right)$ with $\xi_{0}=-i \omega(i k)^{-2 / 3}$ and the branch cut is defined such that $\arg (i k)^{1 / 3}=\pi / 6$ when $k$ is real and positive and $-3 \pi / 2<\arg (k)<\pi / 2$. Differentiating (16b) and using the continuity equation (16a) we find

$$
\tilde{U}_{\xi}^{*}=D_{0} \operatorname{Ai}(\xi)+D_{1} \operatorname{Bi}(\xi) .
$$

For large $\xi$ the function $\mathrm{Bi}(\xi)$ grows exponentially and so $D_{1}=0$. Integrating and using (16c) gives

$$
\tilde{U}^{*}=D_{0} \int_{\xi_{0}}^{\xi} \operatorname{Ai}(\xi) d \xi-\tilde{H}^{*} .
$$

Next, let $\xi \rightarrow \infty$ and use (16d). Hence we find

$$
D_{0}=\frac{\tilde{A}^{*}+\tilde{H}^{*}}{\int_{\xi_{0}}^{\infty} \operatorname{Ai}(\xi) d \xi} .
$$

Setting $Z=0$ in (16b) we obtain

$$
\left.\tilde{U}_{z z}^{*}\right|_{z=0}=(i k)^{2 / 3} D_{0} \mathrm{Ai}^{\prime}\left(\xi_{0}\right)=(i k) \tilde{P}^{*} .
$$


Finally eliminating $D_{0}$ and using the interaction law (16e) (and setting $\sigma=\gamma-1 / \bar{C}$ ), the equation for the wall motion (16f) leads to the dispersion relation for non-trivial solutions

$$
D_{a}(\omega, k)\left[1+\frac{\operatorname{Ai}^{\prime}\left(\xi_{0}\right)}{(i k)^{1 / 3} \int_{\xi_{0}}^{\infty} \operatorname{Ai}(\xi) d \xi} \frac{1}{\left(s-\sigma k^{2}\right)}\right]=-\frac{\operatorname{Ai}^{\prime}\left(\xi_{0}\right)}{(i k)^{1 / 3} \int_{\xi_{0}}^{\infty} \operatorname{Ai}(\xi) d \xi}
$$

For rigid walls the dispersion relation reduces to (see Gajjar (1984))

$$
\frac{\operatorname{Ai}^{\prime}\left(\xi_{0}\right)}{(i k)^{1 / 3} \int_{\xi_{0}}^{\infty} \operatorname{Ai}(\xi) d \xi}=-\left[s-\sigma k^{2}\right]
$$

Expressions similar to (17) for have been derived and discussed before by Mackerrell (1988) for the boundary-layer flow over flexible surfaces and also Walker et al (2006) for supersonic boundary-layer flow over flexible surfaces.

\subsection{Properties of dispersion relation}

For given wall properties, the dispersion relation depends on many parameters $C_{M}, B_{M}, T_{E}, K_{E}, s, \sigma$ and so an exhaustive treatment of the effect of all the different properties is not possible here. However, a few general results can be deduced. Below we have additionally set $\sigma=1$ in the numerical results.

\subsection{Tollmien-Schlichting modes}

There are modes which exist even without wall flexibility and we refer to these as TollmienSchlichting (TS) modes since the properties of these modes are analogous to TS modes in boundary-layer flows. Note that for $k$ small, at leading order the roots of (17) satisfy $\operatorname{Ai}^{\prime}\left(\xi_{0}\right)=0$. The derivative of the Airy function $\operatorname{Ai}(\xi)$ has infinitely many zeros along the negative real axis at locations $\xi=a_{j}^{\prime}$, with $j=1,2, \ldots$ The first few values can be found in many places, with $a_{1}=-1.01879, a_{2}=-3.2482, a_{3}=-4.8201, a_{4}=-6.1633$ see Walker et al (2006) for instance. For large $j$ it is known that (see Abramovitz \& Stegun (1964)),

$$
a_{j}^{\prime} \sim-\left(\frac{3 \pi(4 j-3)}{8}\right)^{\frac{2}{3}}
$$

Using the definition of $\xi_{0}$ we obtain

$$
\omega \sim e^{-\frac{i \pi}{6}} k^{2 / 3}\left|a_{j}^{\prime}\right| \quad \text { for } \quad k \rightarrow 0 .
$$

Thus the roots are clustered along the $\operatorname{ray} \arg (k)=-\pi / 6$ for $k$ positive and $\arg (k)=-5 \pi / 6$ for $k$ negative. Since $\Im(\omega)<0$ this implies stability for small $k$.

For large $k$, the dispersion relation is satisfied by the roots $\xi_{0}=d_{j}$ where $d_{j}$ are the zeros of the integral

$$
\int_{d_{j}}^{\infty} \operatorname{Ai}(\xi) d \xi=0
$$




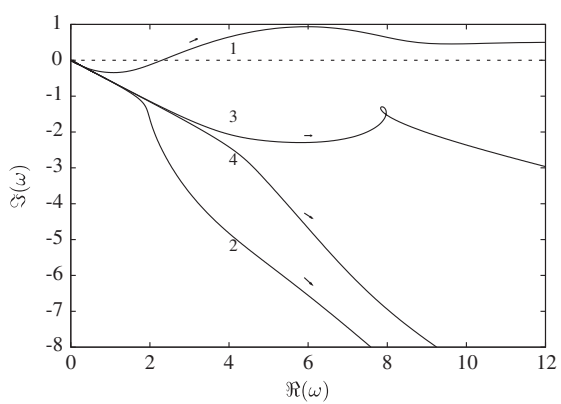

(a)

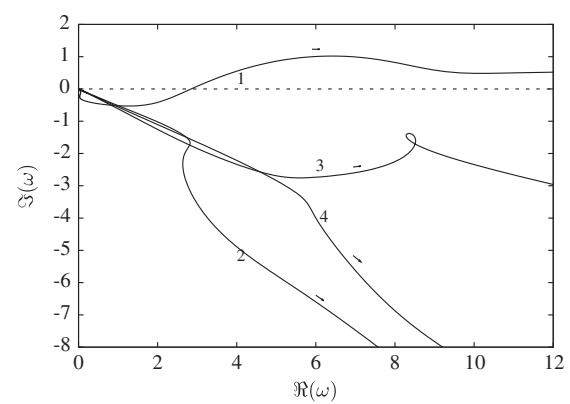

(b)

Figure 3. First four roots from the dispersion relationship for the rigid wall case with (a) $s=0$ and (b) $s=1$ and taking $\sigma=1$. Arrows denote the direction of increasing $k$.

The zeros occur as complex conjugate pairs with negative real part and some values are tabulated in Walker et al (2006). In fact $d_{1}=-4.107-1.1441 i, d_{2}=-4.107+1.441 i, d_{3}=-6.7981-$ $1.0351 i, d_{4}=-6.7981+1.0351 i$. Using the definition of $\xi_{0}$ this gives

$$
\omega \sim d_{j} e^{\frac{5 i \pi}{6}} k^{\frac{2}{3}} \text { for }|k| \gg 1 .
$$

These again correspond to stable modes.

For large $k$ there is an unstable TS mode as well. This has the asymptotic behaviour

$$
\omega \sim \sigma k^{3}-s k-\sigma^{-\frac{1}{2}} e^{\frac{5 i \pi}{4}} k^{-\frac{1}{2}}+\ldots \quad k \rightarrow \infty .
$$

This gives

$$
\Im(\omega) \sim \frac{1}{\sqrt{2 \sigma k}} \quad \text { as } \quad k \rightarrow \infty
$$

and hence instability for large $k$.

\subsection{Modes induced by wall flexibility}

The flexible wall introduces additional modes not present in the rigid-wall case. Some properties of these may also be deduced. For small $k$ it is found numerically that $\omega$ approaches a finite value. From the definition of $\xi_{0}$ this implies that $\xi_{0}$ is large. Hence using the properties of the Airy function and noting that for $\left|\xi_{0}\right|$ large

$$
\frac{\operatorname{Ai}^{\prime}\left(\xi_{0}\right)}{\int_{\xi_{0}}^{\infty} \operatorname{Ai}(\xi) d \xi} \sim-\xi_{0}-\xi_{0}^{-1 / 2}+\ldots,
$$

we can express

$$
\omega=\tilde{\omega}_{0}+\tilde{\omega}_{1} k+\ldots, \quad \text { as } \quad k \rightarrow 0,
$$

with

$$
\tilde{\omega}_{0}=\sqrt{\frac{K_{E}+s}{C_{M}}}, \quad \tilde{\omega}_{1}=\frac{s^{2}}{s(1-s)-2 C_{M} \tilde{\omega}_{0}^{2}} .
$$


For large $k$ another possibility is that corresponding to the inviscid modes induced by the compliant wall, given by setting $D_{a}(\omega, k)=0$ and solving for $\omega$. Using (20) we can solve asymptotically for $\omega$ for $k$ large to obtain

$$
\omega \sim \omega_{0} k^{2}+\omega_{1}+\frac{\omega_{2}}{k}+\frac{\omega_{3}}{k^{2}}+\frac{\omega_{4}}{k^{3}}+\ldots, \quad k \rightarrow \infty .
$$

The dispersion relationship gives

$$
\begin{gathered}
\omega_{0}=\sqrt{\frac{B_{M}}{C_{m}}}, \quad \omega_{1}=\frac{T_{E}}{2 C_{M} \omega_{0}}, \quad \omega_{2}=\frac{1}{2 C_{M}}, \\
\omega_{3}=\frac{\left(\omega_{0}^{2}-\omega_{1}^{2} \sigma C_{M}\right)}{2 C_{M} \sigma \omega_{0}}, \quad \omega_{4}=\frac{1}{2 C_{M}}\left(\frac{\omega_{0}^{3}}{\sigma^{2}}+\omega_{1}+i e^{3 i \pi 4} \omega_{0}^{-\frac{1}{2}}\right)-\frac{\omega_{1} \omega_{2}}{\omega_{0}} .
\end{gathered}
$$

Hence

$$
\Im(\omega) \sim \frac{-1}{2 \sqrt{2 \omega_{0}} C_{M} k^{3}} \quad \text { as } \quad k \rightarrow \infty
$$

and is negative implying stability. Damping has been neglected in the wall model, but if damping is included this destabilises the above mode, as has been noted by Carpenter \& Garrad (1985).

Depending on the various parameters there is also a cross-over between the unstable TS mode (19) and (23).

\subsection{Numerical solution of the dispersion relationship}

The dispersion relationship (17) was solved numerically to obtain $\omega=\omega(k)$. The numerical solutions have the property that if for $k>0,(\omega, k)$ is a root, then so is $(-\bar{\omega},-k)$ where the overbar denotes the complex conjugate, and so only the roots for $k>0$ are shown. The results for the rigid modes are shown in figure 3 . For the rigid case there are no modes induced by the compliant wall, and it can be seen that there is one mode labelled ' 1 ' which becomes unstable for increasing $k$ with the asymptotic form (19). For small $k$ the roots labelled 1,2,3,4 match with the asymptotic form stemming from the root $\xi_{0}=a_{j}^{\prime}, j=1,2,3$, 4. All the other modes are stable. For large $k$ the roots labelled 2,3,4 match with the asymptotic behaviour of the roots

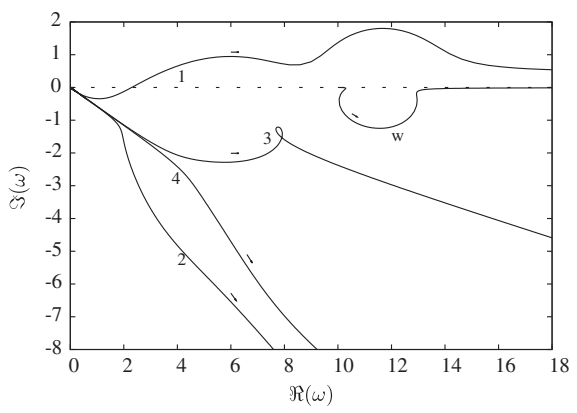

(a)

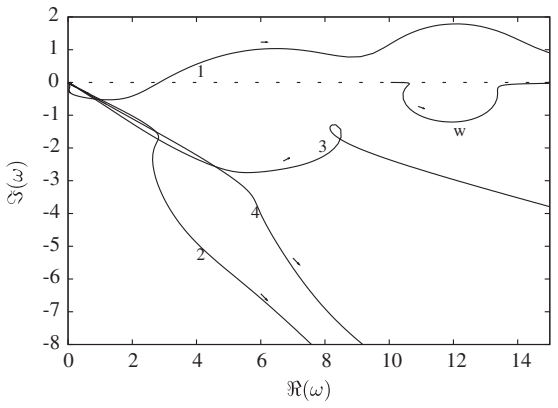

(b)

Figure 4. First four roots from the dispersion relationship for the case with (a) $s=0$ and (b) $s=1$ and taking $\sigma=1$. Also $C_{M}=B_{M}=T_{E}=1$ and $K_{E}=100$. 


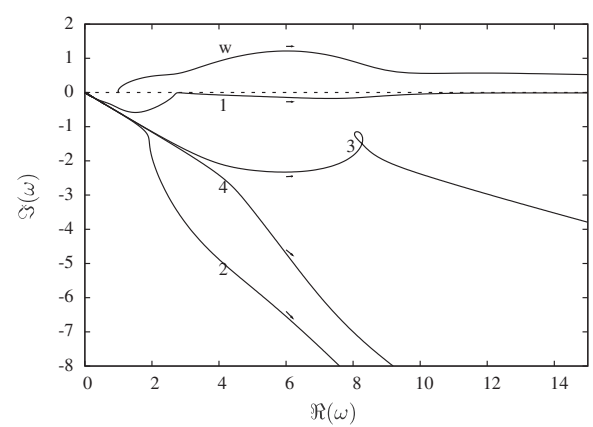

(a)

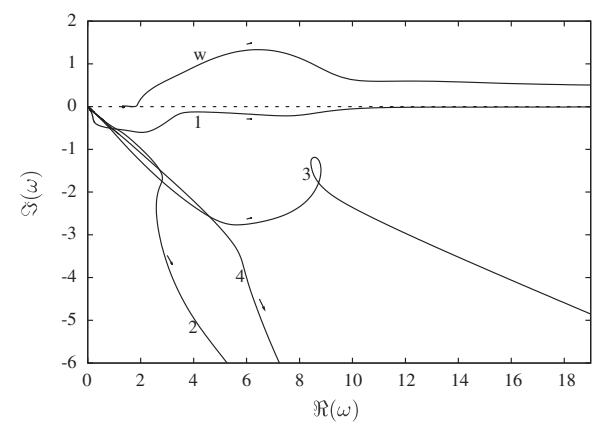

(b)

Figure 5. First four roots from the dispersion relationship for the case with (a) $s=0$ and (b) $s=1$ and taking $\sigma=1$. Also $C_{M}=B_{M}=K_{E}=T_{E}=1$.

stemming from the zeros of $\int_{\xi_{0}}^{\infty} A i(\xi) d \xi=0$. It can be seen that for increasing $s$ the neutral values increase, as noted also by Gajjar (1984).

In figure 4 we present results for $s=0$ and $s=1$ for the case when $C_{M}=B_{M}=T_{E}=1$ with $K_{E}=100$. Since the stiffness is taken to be large, the results shown for the Tollmien-Schlichting modes (labelled 1-4) are not too dissimilar to the results for the rigid wall case in figure 3 . Wall compliance introduces additional modes, and one is shown by the label 'w'. For small values of $k$ the real part of $\omega$ approaches the limit in (22) discussed above and the imaginary part of $\omega$ is very small. For large $k$ the behaviour matches the asymptote (19).

In figure 5, we have shown the results for the case when $s=0$ and $s=1$ with the wall parameters all set equal to one. This corresponds to a more flexible wall.

It can be seen that the wall flexibility in this case stabilises the TS mode 1 with greatly reduced growth rate. On the other hand, the 'w' mode is now destabilised.

\section{Conclusions}

We have formulated the problem for the unsteady separated liquid layer flow induced by a wall distortion and a flexible wall. The linearised stability properties for a flat wall has been examined and it is found that there is a rich set of modes, most of which are stable, but unstable modes also exist. As found in many other studies, the unstable TS mode is stabilised by wall flexibility.

The current study provides a foundation for studying the more interesting problem of a separated flow encountering a region in which the wall is flexible. This however requires a numerical solution of the full nonlinear unsteady triple-deck equations which is currently in progress. Solution of this problem will provide some insight into how a separated boundary-layer flow is affected by a compliant panel.

In the current work, the scalings used stability properties studied are those most relevant to lower-branch modes. The scalings for upper-branch modes, and for travelling wave flutter modes are different. For flat plate boundary-layer flows, these have been discussed in (Carpenter \& Gajjar 1990). Since the upper-branch modes have typically much shorter wavelengths as compared to the lower-branch modes, the latter are expected to be the more important as far as the separated flow is concerned. However, short wavelength instabilities in the vicinity of the separating bubble streamline may be important. 


\section{References}

Abramovitz M and Stegun I 1964 Handbook of mathematical functions with formulas, graphs, and mathematical tables. USA: Dover publications (also available at http://people.math.sfu.ca/ cbm/aands/)

Benjamin T B 1957 Wave formation in laminar flow down and inclined plane. J. Fluid Mech. 2: 554-573

Carpenter P W and Gajjar J S B 1990 A general theory for two- and three-dimensional wall-mode instabilities in boundary layer flow over isotropic and anisotropic compliant walls. Theo. Comp. Fluid Dyn. 1: 349-378

Carpenter P W and Garrad A D 1985 The hydrodynamic stability of flow over Kramer type compliant surfaces. Part I. J. Fluid Mech. 155: 465-510

Gajjar J 1984 On some viscous-inviscid interaction problems in incompressible fluid flows. PhD thesis, Imperial College, London

Gajjar J S B 1987 Fully developed free surface flows - Liquid layer flow over a convex corner. Computers and Fluids 15: 337-360

Logue R P 2008 Stability and bifurcations governed by the triple-deck and related equations. PhD thesis, University of Manchester

Mackerrell S O 1988 Some hydrodynamic instabilities of boundary layer flows. PhD thesis, University of Exeter

Smith F T 1977 Upstream interactions in channel flows. J. Fluid Mech. 1979: 631-655

Walker J D A, Fletcher A and Ruban A I 2006 Instabilities of a flexible surface in supersonic flow. Q.J.M.A.M 59: 253-276

Yih C S 1955 Stability of parallel laminar flow with a free-surface. In Proceedings 2nd US Nat. Congress of Applied Mechanics, ASME, pp. 623-628

Yih C S 1963 Stability of liquid flow down an inclined plane. Physics Fluids 6(3): 321-334

Yih C S 1965 Dynamics of non-homegenous fluids. Macmillan: London 